\title{
The Linked Person Record: Managing the Cancer System through Data Integration
}

\author{
Darren Edery
}

$\mathrm{n}$ all data-rich industries, effective data management is essential if users and stakeholders are to expect value from corporate data. For market-driven enterprises, clean and readily accessible data is vital for maintaining a competitive edge. Quality, integrated data in comprehensive and targeted reports enables effective strategic and tactical decision-making, improves data security, increases the effectiveness of marketing campaigns, ensures tracking of products and services and helps meet regulator demands accurately and with minimal effort. In addition to these strategic benefits, high-performance access to the same data is essential for meeting operational demands from front-line staff at call centres, distribution hubs and network monitoring stations.

In healthcare, data-related priorities are radically different. Marketplace return on investment gives way to improving healthcare delivery, supporting performance management, research and funding analysis. Notwithstanding differences in business priorities, technologies, solutions and methodologies can be intelligently adapted from profit-driven industries to healthcare needs. The Linked Person Record for Cancer, developed for Cancer Care Ontario in 2007-2008, represents the kind of innovation required if healthcare information is to take advantage of advances in information management.

\section{Cancer Care Ontario}

Cancer Care Ontario (CCO) is the provincial agency responsible for improving cancer services. As the government's cancer advisor, CCO directs over $\$ 600$ million in public funding for
The Linked Person Record for Cancer, developed for Cancer Care Ontario in 20072008 , represents the kind of innovation required if healthcare information is to take advantage of advances in information management.

cancer prevention, detection and care.

$\mathrm{CCO}$ has long recognized that information management, performance management and business intelligence are vital to fulfilling its mandate. Since launching its first data warehouse in 2004, the organization has remained dedicated to developing and maintaining cutting-edge data management systems.

The first implementation of the enterprise data warehouse (EDW) consisted of a cancer surveillance data mart to track regional and provincial rates of cancer incidence, mortality and survival for the 20 most common cancers. Subsequent initiatives added data marts to track cancer treatment, drug funding, pathology reports and other related information. A web-based interface, iPort ${ }^{\mathrm{TM}}$, was developed as a highly secure and userfriendly gateway to critical cancer reporting information. In its first three years, the system's user base expanded to over 400 cancer planners, managers and clinicians, establishing iPort ${ }^{\mathrm{TM}}$ as a critical planning tool and a recognized brand within the cancer services community.

As the EDW program entered its fourth development phase 
in August 2006, it met a serious challenge: users were unable to create integrated reporting across the various data marts. As a result, CCO could not provide a comprehensive view of an individual's journey across the cancer care continuum in the EDW. Different data sources had to be linked through custom analyses to allow cancer planners, managers and clinicians to assess clinical quality, understand service utilization and forecast service requirements.

\section{Solution Overview}

Expertise in data warehousing and master data management Record for Cancer in North America. The objective was to create a master patient record, within the EDW, to uniquely identify each cancer patient across various encounters with the healthcare system. Using this master record as an anchor, individual information fragments about cancer patients arriving from disparate data sources can be linked, further enriching the master patient record and enabling tracking and reporting at all levels. After all incoming data is cleansed, standardized and enriched using reliable sources, a sophisticated combination of deterministic and probabilistic linking algorithms match patient records to the master record groups. In addition to automated matching of patient data, the solution aims to allow CCO staff to review matches labelled as incomplete and then manually associate patient records to the correct master record through an easy-to-use web-based interface. Of vital importance - to protect patient privacy, linked records are aggregate views; while the data is linked, the patient cannot be identified. services was brought on board to build the first Linked Person

ability to provide an integrated view to the data consumers who make or guide these types of decisions.

Prior to the implementation of the Linked Person Record for Cancer, CCO's data consumers ran disparate reports from various data marts. Analysis required extensive manual integra-

\section{Expertise in data warehousing and master data management services was brought on board to build the first Linked Person Record for Cancer in North America. The objective was to create a master patient record, within the EDW, to uniquely identify each cancer patient across various encounters with the healthcare system.}

tion work by analysts and statisticians using ad hoc methods and statistical software packages such as SAS and SPSS.

Once the Linked Person Record for Cancer is fully implemented, planners, managers and clinicians will have access to integrated reports that provide aggregated information already linked at the record level. This will facilitate an understanding of how cancer patients move across the continuum of care, while fully protecting the privacy of individual health information. For example, a cancer planner interested in where new treatment facilities will be required must first understand the sociodemographics of a population and the types of cancer they are being diagnosed with, as well as the relationship between where people live and where they obtain treatment. This type of analysis helps planners determine if the incidence of cancer in a specific geographic region is rising more quickly than the regional availability of services appropriate to treatment. The information can also be used to support funding authorities in determining which regions will be most in need of investment in cancer treatment centres.

\section{Business Impact}

Tracking regional and provincial cancer incidence, prevalence, mortality and survival along with linking this information with diagnostic, screening and treatment activity are critical to cancer services planning. Critical decisions on human resources, funding, capital planning and guidelines in evidence-based care, as well as quality and performance monitoring, rely on CCO's
₹ADASTRA 
services in that area may be required. The Linked Person Record for Cancer will facilitate this type of reporting.

\section{A Model for Future Healthcare Solutions}

Data integration is vital to the success of healthcare planning and management. Just as the electronic health record (EHR) has proven essential for healthcare facilities striving to deliver effec-

\section{This type of analysis helps planners} determine if the incidence of cancer in a specific geographic region is rising more quickly than the regional availability of services appropriate to treatment. The information can also be used to support funding authorities in determining which regions will be most in need of investment in cancer treatment centres.

tive care, the linked person record can empower those engaged in analytical and forecasting work. Such technology mitigates the need for guesswork in healthcare planning and can allow cancer system managers to focus their interventions by effectively targeting those areas most in need.

Profit-driven industries have realized the benefit of data integration, and customers, producers and distributors have come to rely on these technologies. In order to provide effective financial guidance, banks require a full view of their customers, who in turn demand an integrated view of their accounts and investments to enable planning and to maximize returns. Telecommunications providers also need to know their customers as fully as possible in order to effectively market new services, and customers increasingly demand flexible packaged services that require central management. In a global marketplace increasingly dependent on just-in-time delivery, retail and distribution networks require integrated views of inventory and sales, without which service and profits would suffer.

While the benefits of data integration for healthcare are selfevident, the industry has faced significant obstacles to successful data integration implementations. Healthcare data is most often housed in non-standardized and disparate sources, with different standards of quality and completeness and irregular delivery schedules. Whereas banks and other industries maintain control over mostly internal data sources, healthcare organizations - especially umbrella organizations such as CCO - rely almost entirely on external sources (hospitals, labs, treatment facilities, etc.) over which they have no direct control. Add to these challenges the sensitivity of healthcare information, and it is no wonder the industry has been slow to fully adopt master data management (MDM).

The innovation of the linked person record solution lies in the approach. Using a series of steps, the team addressed and overcame MDM challenges specific to healthcare. Initially, it was important to understand the data. By combining data profiling, gap analysis and business analysis, the team developed an increased understanding of the data flowing into CCO. Based on this understanding, the next step was to create data cleansing and standardization procedures to align the data. Then, the data was enriched using a high-quality and reliable external source (in this case, provincial health insurance information). The final critical step was to apply a sophisticated combination of rule-based and statistical algorithms to match incoming patient record fragments. These fragment groups were then processed according to business rules, and the best, richest and most reliable information was combined into aggregate composite master person records representing actual cancer patients without identifying actual individuals. The master person records were subsequently loaded into the data warehouse to be used as a reference for the full information continuum.

The success of this implementation is due to several factors: CCO's commitment to improving its reporting capabilities, a well-developed in-house core of experienced subject matter experts with deep knowledge of cancer and healthcare data standards, and Adastra's expertise and creativity. Team members combined their rich MDM experience from other industries with their healthcare information management practices to tackle CCO's data integration challenges.

$\mathrm{CCO}$ is leading the way in leveraging electronic tools for data integration and MDM in the healthcare industry. As the first such solution for cancer data in North America, the Linked Person Record for Cancer is positioned to become an industryleading example of a successful data integration implementation. The commitment to data integration at Cancer Care Ontario has resulted in a more holistic view of the individual journey through the healthcare system and a better understanding of the patient experience. Thus, data integration strategies such as the Linked Person Record for Cancer not only humanize the healthcare system, they also help support better decision-making to ensure that healthcare dollars are spent in the most effective way.

\section{About the Author \\ Darren Edery is Adastra Canada's CEO. Adastra, a global provider of data warehousing, business intelligence and master data management services, worked with Cancer Care Ontario to build the first Linked Person Record for Cancer in North America.}

